\title{
Lifestyle factors and mortality risk in individuals with diabetes mellitus: are the associations different from those in individuals without diabetes?
}

\author{
Diewertje Sluik • Heiner Boeing • Kuanrong Li • Rudolf Kaaks • Nina Føns Johnsen • \\ Anne Tjønneland - Larraitz Arriola • Aurelio Barricarte • Giovanna Masala • Sara Grioni • \\ Rosario Tumino • Fulvio Ricceri • Amalia Mattiello • Annemieke M. W. Spijkerman • \\ Daphne L. van der A • Ivonne Sluijs • Paul W. Franks • Peter M. Nilsson • \\ Marju Orho-Melander • Eva Fhärm • Olov Rolandsson • Elio Riboli • Dora Romaguera • \\ Elisabete Weiderpass • Emilio Sánchez-Cantalejo • Ute Nöthlings
}

Received: 22 April 2013 / Accepted: 9 September 2013 / Published online: 17 October 2013

(C) Springer-Verlag Berlin Heidelberg 2013

\begin{abstract}
Aims/hypothesis Thus far, it is unclear whether lifestyle recommendations for people with diabetes should be different from those for the general public. We investigated whether the associations between lifestyle factors and mortality risk differ between individuals with and without diabetes.

Methods Within the European Prospective Investigation into Cancer and Nutrition (EPIC), a cohort was formed of 6,384
\end{abstract}

D. Sluik $\cdot$ H. Boeing $\cdot$ U. Nöthlings

Department of Epidemiology, German Institute of Human Nutrition

Potsdam-Rehbruecke, Nuthetal, Germany

D. Sluik $(\bowtie)$

Division of Human Nutrition, Wageningen University,

P.O. Box 8129,6700 , EV Wageningen, the Netherlands

e-mail: Diewertje.Sluik@wur.nl

K. Li • R. Kaaks

Division of Cancer Epidemiology, German Cancer Research Centre,

Heidelberg, Germany

N. F. Johnsen • A. Tjønneland

Danish Cancer Society, Copenhagen, Denmark

L. Arriola

Public Health Division of Gipuzkoa BIO-Donostia Basque

Government, San Sebastian, Spain

L. Arriola $\cdot$ A. Barricarte $\cdot$ E. Sánchez-Cantalejo

Consortium for Biomedical Research in Epidemiology and Public

Health, Murcia, Spain

A. Barricarte

Navarre Public Health Institute, Pamplona, Spain persons with diabetes and 258,911 EPIC participants without known diabetes. Joint Cox proportional hazard regression models of people with and without diabetes were built for the following lifestyle factors in relation to overall mortality risk: BMI, waist/height ratio, 26 food groups, alcohol consumption, leisure-time physical activity, smoking. Likelihood ratio tests for heterogeneity assessed statistical differences in regression coefficients.

G. Masala

Molecular and Nutritional Epidemiology Unit,

Cancer Research and Prevention Institute, Florence, Italy

S. Grioni

Epidemiology and Prevention Unit, National Cancer Institute, Milan, Italy

\section{R. Tumino}

Cancer Registry and Histopathology Unit,

‘Civile-M.P. Arezzo’ Hospital, Ragusa, Italy

F. Ricceri

Human Genetics Foundation, Turin, Italy

A. Mattiello

Department of Clinical and Experimental Medicine,

Federico II University, Naples, Italy

A. M. W. Spijkerman

Centre for Prevention and Health Services Research,

National Institute for Public Health and the Environment,

Bilthoven, the Netherlands 
Results Multivariable adjusted mortality risk among individuals with diabetes compared with those without was increased, with an HR of 1.62 (95\% CI 1.51, 1.75). Intake of fruit, legumes, nuts, seeds, pasta, poultry and vegetable oil was related to a lower mortality risk, and intake of butter and margarine was related to an increased mortality risk. These associations were significantly different in magnitude from those in diabetes-free individuals, but directions were similar. No differences between people with and without diabetes were detected for the other lifestyle factors. Conclusions/interpretation Diabetes status did not substantially influence the associations between lifestyle and mortality risk. People with diabetes may benefit more from a healthy diet, but the directions of association were similar. Thus, our study suggests that lifestyle advice with respect to mortality for patients with diabetes should not differ from recommendations for the general population.

Keywords Adiposity $\cdot$ Alcohol $\cdot$ Diabetes $\cdot$ Lifestyle $\cdot$ Mortality $\cdot$ Nutrition $\cdot$ Physical activity $\cdot$ Smoking

\section{Abbreviations \\ CVD Cardiovascular disease \\ EPIC European Prospective Investigation into Cancer and Nutrition \\ FFQ Food frequency questionnaire}

D. L. van der A

National Institute for Public Health and the Environment, Bilthoven, the Netherlands

I. Sluijs

Julius Centre for Health Sciences and Primary Care, University

Medical Centre, Utrecht, the Netherlands

P. W. Franks

Department of Clinical Sciences, Genetic and Molecular Epidemiology Unit, Skåne University Hospital, Lund University, Malmö, Sweden

P. W. Franks

Department of Nutrition, Harvard School of Public Health,

Boston, MA, USA

\section{P. M. Nilsson}

Department of Clinical Sciences, Lund University,

University Hospital, Malmö, Sweden

\section{Orho-Melander}

Department of Clinical Sciences, Diabetes and Cardiovascular

Disease - Genetic Epidemiology, Lund University,

Malmö, Sweden

\section{E. Fhärm}

Family Medicine, Institution of Public Health and Clinical Medicine, Umeå University, Umeå, Sweden

\section{O. Rolandsson}

Department of Public Health and Clinical Medicine,

Family Medicine, Umeå University, Umeå, Sweden

\section{Introduction}

Individuals with diabetes mellitus have an increased risk of cardiovascular disease (CVD) and premature mortality $[1,2]$. It has been estimated that diabetes confers a 1.8 -fold increased risk of mortality [3]. A major objective of diabetes management is to prevent or minimise micro- and macrovascular complications, typically through lifestyle modifications and pharmacotherapy $[4,5]$, the former of which usually emphasises reducing body weight (or maintaining a healthy body weight), regular physical activity, moderate alcohol consumption and a healthy diet $[5,6]$. The evidence supporting these recommendations has rarely been derived from studies of people with diabetes $[5,7]$.

To strengthen the evidence base for public health decisionmaking, we have previously investigated associations between selected lifestyle factors and mortality in a large European cohort of individuals with prevalent diabetes. Measures of abdominal adiposity, but not general adiposity, were found to be associated with higher mortality risk [8]. Furthermore, our results on alcohol consumption support the current international recommendation that people with diabetes can consume alcohol within the recommended upper limits [9]. Moreover, people with diabetes who undertake moderate amounts of physical activity are at appreciably lower risk of death than inactive persons [10].

E. Riboli $\cdot$ D. Romaguera

School of Public Health, Imperial College London, London, UK

D. Romaguera

CIBER Fisiopatología de la Obesidad y Nutrición (CIBER-OBN), Spain

URL: www.ciberobn.es

E. Weiderpass

Department of Community Medicine, Faculty of Health Sciences, University of Tromsø, The Arctic University of Norway, Tromsø, Norway

E. Weiderpass

Department of Research, Cancer Registry of Norway, Oslo, Norway

E. Weiderpass

Department of Medical Epidemiology and Biostatistics, Karolinska Institutet, Stockholm, Sweden

E. Weiderpass

Samfundet Folkhälsan, Helsinki, Finland

E. Sánchez-Cantalejo

Andalusian School of Public Health, Granada, Spain

U. Nöthlings

Epidemiology Section, Institute for Experimental Medicine, Christian-Albrechts-University of Kiel, Kiel, Germany

U. Nöthlings

Institute for Nutrition and Food Science, University of Bonn,

Bonn, Germany 
Several other studies have also investigated the relationship between lifestyle and mortality in individuals with diabetes; moreover, many other studies have systematically excluded persons with diabetes to simplify the analysis, making it unclear how to apply these results to such people. Because diabetes increases mortality risk [11], it has been hypothesised that persons with diabetes should benefit to a greater extent from a healthy lifestyle [5], but no indications were found in the existing literature that associations between lifestyle factors and risk of mortality differ between people with and without diabetes.

Whether lifestyle recommendations should differ between persons with and without diabetes is also of particular interest for individuals living with undiagnosed diabetes today [12] or those with impaired glucose tolerance [13]. The rationale of adopting lifestyle habits in line with the recommendation in this stratum of the population is further supported by the large body of evidence showing CVD risk as well $[13,14]$.

We investigated the associations of BMI, waist/height ratio, 26 food groups, alcohol consumption, physical activity and smoking with overall mortality in individuals with diabetes compared with those without in a large European prospective study. Our aim was to provide empirical evidence relating to whether different healthy lifestyle recommendations for individuals with diabetes and the general public are justified.

\section{Methods}

Study design and population Within the European Prospective Investigation into Cancer and Nutrition (EPIC), which is an ongoing multicentre prospective study in ten European countries [15], a cohort was defined of participants with a confirmed diagnosis of type 1 or type 2 diabetes at baseline. EPIC is a cohort study including 519,978 participants, aged 35-70 years, from 23 centres in ten European countries who were recruited from 1992 to 2000. Participants were predominantly recruited from the general population residing in a certain geographic area (town, province or country). As described previously [8], 15 EPIC study centres from six European countries provided additional data on diabetes diagnosis and medication. Self-reports of diagnosis obtained at baseline were confirmed by additional information sources. The study was conducted according to the guidelines laid down in the Declaration of Helsinki and was approved by the ethics review boards of the single centres and the International Agency for Research on Cancer in Lyon, France. All participants provided written informed consent. The cohort comprised 6,412 individuals with confirmed diabetes at study entry, and, after exclusion of participants without follow-up information on vital status $(n=28)$, the analytical sample included 6,384 individuals with diabetes. Subsequently, 258,911 participants without a verified or a self-reported diabetes diagnosis at baseline from the same EPIC study centres were selected as the group without diabetes. Thus, the analytical sample included 6,384 individuals with and 258,911 individuals without diabetes.

Exposure assessment At baseline, weight and height were measured with participants not wearing shoes. Waist circumference was measured either at the narrowest circumference of the torso or at the midpoint between the lower ribs and iliac crest [8].

Dietary intake during the preceding 12 months was assessed at baseline with country-specific instruments that had been developed. Extensive quantitative dietary questionnaires with up to 300-500 food items, semi-quantitative food frequency questionnaires (FFQs) and combined dietary methods of food records and questionnaires were used [15]. In a series of validation studies in the various source populations, the FFQs had good repeatability, and validity ranged from modest to good [16-19]. In addition, a highly standardised reference dietary measurement was taken from an $8 \%$ age-stratified random sample of the cohort $(n=36,994)$ using a computerised $24 \mathrm{~h}$ dietary recall. Foods were organised into 39 food groups, and intake of 26 meaningful food groups was adjusted for energy with the residual method [20] and analysed as predicted by regression calibration [21].

Alcohol consumption during the preceding 12 months was assessed with the baseline FFQ. Weekly consumption of alcohol retrospectively at the ages of 20,30,40 and 50 years was assessed in the lifestyle questionnaire, except in the study centres at Bilthoven, Naples and Sweden. Previous alcohol consumption was defined as none, always moderate, and sometimes heavy consumption. In our previous study, we have shown that 'light alcohol consumers' is a more appropriate reference group than 'non-consumers'; therefore, an alcohol consumption of $>0-6 \mathrm{~g} /$ day was used as reference [9]. Within EPIC, the amount of alcoholic beverage consumed by comparable age cohorts at certain ages was validated by comparison of self-reported data with the respective per capita measures and showed, in general, good reproducibility [22].

Leisure-time physical activity was assessed with a lifestyle questionnaire at baseline and included information on walking, cycling, gardening, sport, household and do-it-yourself activities; this questionnaire was not validated. Detailed information about smoking was also collected through the lifestyle questionnaire, including smoking status at recruitment and smoking history.

Covariate assessment Educational level and medical history, including prevalent myocardial infarction, stroke and cancer, were also obtained using baseline questionnaires.

For the participants with diabetes, duration since diabetes diagnosis was calculated by subtracting the self-reported year 
of diagnosis or, when available, the exact date of diagnosis supplied by the medical practitioner from the year of baseline examination. Information on insulin therapy or use of oral hypoglycaemic agents was either self-reported at the visit to the study centre or obtained during medical verification. The lifestyle questionnaire also included a question on insulin therapy.

Outcome ascertainment Causes and dates of death were ascertained by several methods, depending on the available options in each country, following a standardised protocol. Record links with local, regional or central cancer registries, boards of health or death registries were used in Denmark, Italy, the Netherlands, Spain and Sweden. Germany identified deceased participants by follow-up mailings to participants and their next of kin and subsequent inquiries to municipality registries, regional health departments, physicians or hospitals.

Statistical analysis Statistical analyses were performed with SAS 9.2 (SAS Institute, Cary, NC, USA). The LIFETEST procedure was used to create a Kaplan-Meier curve and perform a logrank test. All-cause mortality risk for people with diabetes vs those without was estimated with HRs and 95\% CIs using standard Cox proportional hazard regression [23]. Age was used as the underlying time scale, with time of entry defined as the participant's age in years at recruitment, and time of exit defined as the participant's age in years at death or censoring.

To compare associations of lifestyle factors and mortality between persons with and without diabetes, an extension of the Cox proportional hazard model was prepared based on the ideas and methodology of the competing risk model of Lunn and McNeil [24] and a statistical test for the equality of differently adjusted incidence rate ratios devised by Hoffmann et al [25]. In this model, diabetes status was characterised by $\delta=1$, and no known diabetes by $\delta=0$. Product terms of $\delta$ and $(\delta-1)$ were added to allow for differences in the effect size of $X$ and the covariates $Z$ by diabetes status. This resulted in exclusion of the covariates for people with diabetes in the risk estimates for those without diabetes and vice versa, although they were included in the joint model. The hazard function $\lambda(t)$ of the Cox model had the following form:

$\lambda(t)=\lambda_{0}(t) \exp \left\{\beta_{1} \delta X+\beta_{2}(1-\delta) X+\delta \theta^{\mathrm{T}} Z+(1-\delta) \theta^{T} Z\right\}$

where $\lambda_{0}(t)$ was the unspecified baseline hazard function, $\beta_{1}$ the parameter estimate in diabetic patients, $\beta_{2}$ the parameter estimate in non-diabetic individuals, and $Z$ a vector of covariates.

Robust sandwich estimates for the covariance matrix were not calculated, since the observations were not dependent-in contrast with competing risk analyses. Diabetes status was entered as a stratum variable to allow the baseline hazard functions to have no constant ratio. Moreover, this ensures that the HR for the two groups are identical with those obtained when two separate proportional hazards regression models are used. Subsequently, likelihood ratio tests for heterogeneity were used to quantify differences in $\beta_{1}$ and $\beta_{2}$ for all studied lifestyle factors. The hypothesis for this test states that the two HRs for persons with and without diabetes are equal [25]. In addition, these results from the joint Cox models were compared with a test for interaction. Statistical interaction for diabetes status was tested by adding product terms (exposure $\times$ diabetes status) to the standard Cox regression model in the whole sample, also including diabetes status as a covariate.

Putative confounding factors were selected using directed acyclic graphs [26]. HRs were stratified for age and centre and adjusted for sex, self-reported myocardial infarction, stroke or cancer at baseline, educational attainment (none, primary school, secondary school, technical or professional school, or higher), alcohol consumption (grams per day), physical activity (inactive, moderately inactive, moderately active or active), smoking status (never, former [quit $\leq 10$ years ago, 11 20 years ago, $>20$ years ago] or current), smoking duration ( $\leq 10$ years, $11-20$ years, $21-30$ years, $31-40$ years, $\geq 41$ years) and smoking intensity $(<15,15-24$ or $>25$ cigarettes smoked daily), and the underlying dietary patterns when there were no exposure variables. To take diet into account, the model was adjusted for the factor loadings for the first three dietary patterns derived from factor analysis on 26 food groups. In participants with diabetes, HRs were additionally adjusted for disease duration (years) and use of diabetes medication (none, insulin, oral hypoglycaemic agents, or both).

Proportions of missing data were by and large similar between persons with and without diabetes and were $<1 \%$ for BMI, $6-11 \%$ for waist/height ratio, $2-3 \%$ for diet, $<1 \%$ for alcohol consumption, $6-12 \%$ for physical activity, $<1 \%$ for smoking status, $<1 \%$ for education, $1-2 \%$ for prevalent myocardial infarction, $6-11 \%$ for prevalent stroke and $10-15 \%$ for prevalent cancer. Among the participants with diabetes, $29 \%$ had missing data on medication and $9 \%$ on disease duration. These missing values were imputed with the multiple imputation technique [27] in which 20 duplicate datasets were sampled, with the missing values replaced by imputed values $[28,29]$.

The robustness of the results was investigated by first excluding those with comorbidities at baseline or a followup of $<2$ years. Second, since people with diabetes may be more likely to under-report their energy intake than those without diabetes, it was checked whether excluding energy misreporters (those who were in the top and bottom $1 \%$ of the ratio of energy intake to energy requirement) influenced the results on food groups (177 diabetic and 5,023 non-diabetic participants excluded). 


\section{Results}

After a median follow-up of 9.9 years (interquartile range 8.8-11.0 years), 830 (13\%) participants with diabetes and 12,135 (5\%) participants without known diabetes had died. Compared with participants without diabetes, those with diabetes were older, more likely to be male and had a higher BMI and waist/height ratio (Table 1). Those with diabetes had lower alcohol consumption and were more likely to be physically inactive and educated to a lower standard than their nondiabetic counterparts. In addition, prevalence of hypertension and heart disease were higher among those with diabetes.

The individuals with diabetes had significantly worse 12-year survival rates than those without (Fig. 1). Moreover, all-cause mortality risk among those with diabetes with respect to those without was HR 1.62 (95\% CI 1.51, 1.75) after adjustment for age, sex, prevalent diseases, educational status, BMI, waist/height ratio, diet, alcohol consumption, physical activity and smoking behaviour.

Table 2 shows the HR for overall mortality from a joint model of people with and without diabetes as well as the ratio between the HR and $p$ value for difference. A higher BMI and waist/height ratio were related to increased mortality in diabetes-free individuals. These associations tended to be stronger in those with diabetes, although the difference was not statistically significant.

The comparison of mortality risks by intake of 26 food groups or items showed several statistically significant differences between individuals with and without diabetes. A higher consumption of fruit, legumes, nuts and seeds, pasta, poultry and vegetable oil was associated with a lower mortality risk in individuals with diabetes. Moreover, a higher consumption of butter and margarine was related to a higher mortality risk in individuals with diabetes. Unexpectedly, cakes and cookies and soft drinks were inversely related to mortality in diabetes-free individuals.

Compared with light alcohol consumption ( $>0-6 \mathrm{~g} /$ day), abstinence and high consumption ( $>60 \mathrm{~g} /$ day) of alcohol were associated with increased mortality risk. Moreover, compared with light alcohol consumption, a consumption of 6-60 g/day was associated with a lower mortality risk in persons without diabetes, but not in persons with diabetes. However, these differences were not statistically significant.

Higher amounts of leisure-time physical activity were related to lower mortality rates in persons with and without diabetes, but again these differences were not statistically significant.

Finally, former and current smokers had an increased mortality risk compared with never smokers irrespective of diabetes status.

With the exception of the results for waist/height ratio, the $p$ values from the test for statistical interaction by diabetes status in standard Cox regression models were similar to the
Table 1 General characteristics of 6,384 persons with diabetes and 258, 911 persons without diabetes from the EPIC cohort

\begin{tabular}{|c|c|c|}
\hline Characteristic & Diabetic $(n=6,384)$ & $\begin{array}{l}\text { Non-diabetic } \\
(n=258,911)\end{array}$ \\
\hline Age (years) & $57.4(6.7)$ & $51.8(9.2)$ \\
\hline Male (\%) & 54 & 40 \\
\hline BMI $\left(\mathrm{kg} / \mathrm{m}^{2}\right)$ & $28.9(4.9)$ & $25.8(4.1)$ \\
\hline Waist/height ratio & $0.58(0.08)$ & $0.51(0.07)$ \\
\hline \multicolumn{3}{|l|}{ Food group intake (g/day) } \\
\hline Potatoes & $85(60-116)$ & $76(50-104)$ \\
\hline Vegetables & $157(129-192)$ & $147(118-180)$ \\
\hline Fruit & $193(133-281)$ & $182(121-277)$ \\
\hline Legumes & $3(0-7)$ & $2(0-6)$ \\
\hline Nuts and seeds & $1(0-2)$ & $2(0-4)$ \\
\hline \multicolumn{3}{|l|}{ Dairy } \\
\hline Milk and milk products & $137(64-238)$ & $149(75-243)$ \\
\hline Cheese & $31(24-39)$ & $32(25-41)$ \\
\hline Yogurt & $32(3-71)$ & $39(11-76)$ \\
\hline \multicolumn{3}{|l|}{ Grains } \\
\hline Pasta & $16(8-34)$ & $21(12-44)$ \\
\hline Rice & $10(6-17)$ & $12(8-19)$ \\
\hline Bread & $128(100-164)$ & $121(94-157)$ \\
\hline Breakfast cereals & $0(0-6)$ & $0(0-12)$ \\
\hline \multicolumn{3}{|l|}{ Meat } \\
\hline Red & $50(38-66)$ & $46(34-60)$ \\
\hline Processed & $49(34-70)$ & $41(28-59)$ \\
\hline Poultry & $16(11-23)$ & $15(11-22)$ \\
\hline Offal & $1(0-2)$ & $1(0-2)$ \\
\hline Fish and shellfish & $27(18-41)$ & $27(17-37)$ \\
\hline Eggs & $13(10-18)$ & $13(11-18)$ \\
\hline \multicolumn{3}{|l|}{ Fats and oils } \\
\hline Vegetable oil & $2(1-5)$ & $2(1-6)$ \\
\hline Butter and margarine & $22(7-37)$ & $20(5-33)$ \\
\hline Sugar and confectionery & $27(19-36)$ & $36(28-46)$ \\
\hline Cake and cookies & $42(34-53)$ & $50(40-62)$ \\
\hline \multicolumn{3}{|l|}{ Non-alcoholic beverages } \\
\hline Soft drinks & $9(0-104)$ & $47(0-194)$ \\
\hline Juices & $33(0-96)$ & 43 (13-99) \\
\hline Tea & $26(0-151)$ & $48(0-194)$ \\
\hline Coffee & $487(211-664)$ & $488(173-697)$ \\
\hline Alcohol consumption & $5(1-20)$ & $8(2-20)$ \\
\hline \multicolumn{3}{|c|}{ Leisure-time physical activity (\%) } \\
\hline Low & 26 & 22 \\
\hline Medium & 23 & 21 \\
\hline High & 25 & 23 \\
\hline Very high & 26 & 24 \\
\hline \multicolumn{3}{|l|}{ Smoking status $(\%)$} \\
\hline Never & 39 & 42 \\
\hline Former & 28 & 29 \\
\hline Current & 25 & 28 \\
\hline
\end{tabular}


Table 1 (continued)

\begin{tabular}{lll}
\hline Characteristic & Diabetic $(n=6,384)$ & $\begin{array}{l}\text { Non-diabetic } \\
(n=258,911)\end{array}$ \\
\hline Educational attainment (\%) & & \\
$\quad$ None & 4 & 2 \\
Primary school & 42 & 28 \\
Secondary school & 27 & 28 \\
Technical/professional school & 11 & 20 \\
Higher (including university) & 16 & 21 \\
Comorbidities (\%) & & \\
Hypertension & 55 & 27 \\
Heart disease & 7 & 1 \\
Stroke & 4 & 1 \\
Cancer & 4 & 3
\end{tabular}

Continuous variables are shown as mean (SD) when normally distributed and median (interquartile range) when not normally distributed. Categorical variables are shown as percentages

results from the joint regression models and the test for heterogeneity.

Excluding participants with comorbidities at baseline from sensitivity analyses did not substantially influence risk estimates of persons with and without diabetes. Excluding energy misreporters did not affect the results substantially (data not shown).

\section{Discussion}

The results from this prospective study confirm that people with diabetes have a higher premature mortality risk than those without diabetes [11]. Diabetes status did not substantially affect the associations between mortality risk and adiposity, physical activity, smoking and alcohol consumption. Importantly, intake of several food groups was more strongly related to mortality risk in individuals with diabetes than in

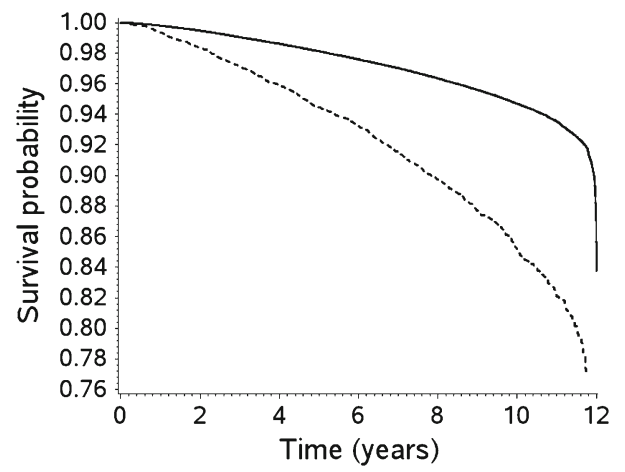

Fig. 1 Twelve-year survival curves for 258,911 participants without diabetes (solid line) vs 6,384 with diabetes (dashed line). $p$ value of logrank test $<0.0001$ those without diabetes, but the directions of association were generally the same.

We found three other studies that have tested whether associations between lifestyle and mortality risk are statistically different in people with and without diabetes. Batty et al [30] explored whether diabetes status modified the effect of physical activity on mortality in 352 men with diabetes and 6,056 normoglycaemic men. The association between walking pace and leisure-time physical activity and CVD outcomes was stronger in men with diabetes ( $p$ value for interaction was statistically significant). In our study, leisure-time physical activity was associated with a lower mortality risk, but the associations were not different from those in persons without diabetes. Conversely, the National Health and Nutrition Examination Survey I reported no statistically significant interaction by diabetes status for the associations between the lifestyle factors, smoking, BMI, physical activity and mortality [31]. Moreover, in our 2011 study of adiposity and mortality, we tested for statistical interaction between measures of adiposity and diabetes status in relation to mortality in the general EPIC population [8]. No statistically significant interaction was detected. In the present study, we found no statistically significant difference between persons with and without diabetes when comparing the associations between adiposity measures and mortality.

To our knowledge, this study is the first to use the methodology of the competing risk model to quantify and test for differences in epidemiological associations between populations. We believe this was the most appropriate approach to answer our study questions. Our results are comparable with those from a 'standard' test of statistical interaction by diabetes status. However, a p value from a test of interaction only gives an indication of whether the relationship is different between two groups. The competing risk approach enabled us to compare and quantify the differences in strength and the direction of associations between persons with and without diabetes.

We have observed several significant associations between lifestyle factors and mortality risk. Higher adiposity measures, smoking and higher levels of alcohol consumption as well as abstinence were related to increased mortality risk. Furthermore, physical activity was inversely associated with mortality. These associations are well established in the scientific literature in individuals both with and without diabetes. This study further adds to this body of evidence that these associations are not affected by diabetes status.

Next, a higher consumption of fruit, legumes, nuts and seeds, pasta, poultry and vegetable oil was associated with a lower mortality risk. Furthermore, a higher consumption of butter and margarine was related to a higher mortality risk. These associations were significantly stronger in persons with diabetes than in their non-diabetic counterparts. We cannot 
Table 2 HR $(95 \%$ CI) for selected lifestyle factors and mortality in 6,384 persons with and 258,911 persons without diabetes and the ratio between these HRs

\begin{tabular}{|c|c|c|c|c|c|}
\hline Lifestyle factor & Diabetic & Non-diabetic & Ratio & $p$ value difference & $p$ value int \\
\hline \multicolumn{6}{|l|}{ Adiposity $^{\mathrm{a}}$} \\
\hline \multicolumn{6}{|l|}{ BMI } \\
\hline Q1 & 1 & 1 & 1 & & \\
\hline Q2 & $0.80(0.61,1.05)$ & $0.84(0.80,0.89)$ & $0.95(0.72,1.25)$ & 0.71 & 0.30 \\
\hline Q3 & $0.78(0.61,1.00)$ & $0.84(0.80,0.89)$ & $0.93(0.72,1.20)$ & 0.56 & 0.16 \\
\hline Q4 & $0.90(0.72,1.13)$ & $1.03(0.97,1.08)$ & $0.88(0.70,1.11)$ & 0.27 & 0.03 \\
\hline Continuous (per $\mathrm{kg} / \mathrm{m}^{2}$ ) & $1.02(1.00,1.03)$ & $1.01(1.01,1.02)$ & $1.01(0.99,1.02)$ & 0.37 & 0.56 \\
\hline \multicolumn{6}{|l|}{ Waist/height ratio } \\
\hline Q1 & 1 & 1 & 1 & & \\
\hline Q2 & $0.73(0.52,1.04)$ & $0.94(0.89,1.00)$ & $0.78(0.55,1.11)$ & 0.16 & 0.03 \\
\hline Q3 & $0.74(0.54,1.01)$ & $0.95(0.89,1.01)$ & $0.78(0.56,1.07)$ & 0.12 & 0.01 \\
\hline Q4 & $0.97(0.73,1.30)$ & $1.22(1.15,1.29)$ & $0.80(0.60,1.08)$ & 0.14 & 0.003 \\
\hline Continuous (per unit) & $9.28(4.78,18.01)$ & $6.83(5.15,9.06)$ & $1.36(0.70,2.64)$ & 0.37 & 0.47 \\
\hline \multicolumn{6}{|l|}{ Food groups $\mathrm{s}^{\mathrm{a}}$} \\
\hline Potatoes (per $100 \mathrm{~g}$ ) & $1.10(0.91,1.32)$ & $0.96(0.90,1.01)$ & $1.15(0.95,1.38)$ & 0.16 & 0.09 \\
\hline Vegetables (per $100 \mathrm{~g}$ ) & $0.74(0.64,0.86)$ & $0.85(0.81,0.90)$ & $0.87(0.75,1.02)$ & 0.08 & 0.05 \\
\hline Fruit (per $100 \mathrm{~g}$ ) & $0.85(0.79,0.92)$ & $0.97(0.95,1.00)$ & $0.88(0.81,0.94)$ & 0.001 & 0.001 \\
\hline Legumes (per $10 \mathrm{~g}$ ) & $0.88(0.81,0.96)$ & $1.03(1.00,1.05)$ & $0.86(0.79,0.94)$ & 0.001 & $<0.0001$ \\
\hline Nuts and seeds (per g) & $0.94(0.90,0.97)$ & $0.99(0.98,1.00)$ & $0.95(0.91,0.98)$ & 0.01 & 0.02 \\
\hline \multicolumn{6}{|l|}{ Dairy } \\
\hline Milk (products) (per $50 \mathrm{~g}$ ) & $1.01(0.99,1.04)$ & $1.01(1.01,1.02)$ & $1.00(0.98,1.02)$ & 0.83 & 0.97 \\
\hline Yogurt (per $10 \mathrm{~g}$ ) & $1.01(1.00,1.02)$ & $1.00(1.00,1.00)$ & $1.01(1.00,1.02)$ & 0.21 & 0.06 \\
\hline Cheese (per $10 \mathrm{~g}$ ) & $0.99(0.94,1.05)$ & $0.96(0.95,0.98)$ & $1.03(0.98,1.09)$ & 0.23 & 0.06 \\
\hline \multicolumn{6}{|l|}{ Grains } \\
\hline Pasta (per $10 \mathrm{~g}$ ) & $0.93(0.90,0.96)$ & $0.99(0.98,1.00)$ & $0.94(0.92,0.98)$ & 0.001 & 0.001 \\
\hline Rice (per 10 g) & $0.93(0.86,1.00)$ & $0.96(0.94,0.98)$ & $0.97(0.90,1.05)$ & 0.46 & 0.40 \\
\hline Bread (per $10 \mathrm{~g}$ ) & $0.87(0.71,1.07)$ & $0.81(0.76,0.82)$ & $1.08(0.88,1.33)$ & 0.47 & 0.38 \\
\hline Breakfast cereals (per $10 \mathrm{~g}$ ) & $1.00(0.97,1.02)$ & $0.97(0.97,0.98)$ & $1.02(1.00,1.05)$ & 0.09 & 0.02 \\
\hline \multicolumn{6}{|l|}{ Meat } \\
\hline Red (per $10 \mathrm{~g}$ ) & $1.00(0.96,1.04)$ & $1.01(1.00,1.02)$ & $0.99(0.96,1.03)$ & 0.73 & 0.29 \\
\hline Processed (per $10 \mathrm{~g}$ ) & $1.03(1.00,1.07)$ & $1.03(1.02,1.04)$ & $1.00(0.97,1.04)$ & 0.86 & 0.80 \\
\hline Poultry (per $10 \mathrm{~g}$ ) & $0.89(0.83,0.96)$ & $0.97(0.95,1.00)$ & $0.91(0.85,0.99)$ & 0.02 & 0.01 \\
\hline Offal (per g) & $1.00(0.97,1.03)$ & $1.01(1.01,1.02)$ & $0.99(0.96,1.01)$ & 0.30 & 0.16 \\
\hline Fish and shellfish (per $10 \mathrm{~g}$ ) & $0.99(0.96,1.02)$ & $1.00(0.96,1.02)$ & $0.99(0.96,1.02)$ & 0.52 & 0.18 \\
\hline Eggs (per $10 \mathrm{~g})$ & $1.04(0.96,1.12)$ & $1.09(1.06,1.12)$ & $0.95(0.88,1.03)$ & 0.21 & 0.09 \\
\hline \multicolumn{6}{|l|}{ Fats } \\
\hline Vegetable oil (per g) & $0.97(0.96,0.98)$ & $0.99(0.98,1.00)$ & $0.98(0.97,0.99)$ & $<0.0001$ & $<0.0001$ \\
\hline Butter and margarine (per g) & $1.05(1.02,1.09)$ & $1.00(0.99,1.02)$ & $1.05(1.01,1.09)$ & 0.01 & 0.004 \\
\hline Sugar and confectionery (per $10 \mathrm{~g}$ ) & $1.06(0.99,1.12)$ & $1.05(1.03,1.07)$ & $1.00(0.94,1.07)$ & 0.89 & 0.81 \\
\hline Cakes and cookies (per $10 \mathrm{~g}$ ) & $1.03(0.99,1.08)$ & $0.97(0.96,0.99)$ & $1.06(1.02,1.10)$ & 0.01 & 0.002 \\
\hline \multicolumn{6}{|l|}{ Non-alcoholic beverages } \\
\hline Soft drinks (per $10 \mathrm{~g}$ ) & $1.04(0.99,1.09)$ & $0.98(0.96,0.99)$ & $1.06(1.01,1.12)$ & 0.02 & 0.01 \\
\hline Juices (per $10 \mathrm{~g}$ ) & $0.98(0.94,1.01)$ & $0.97(0.96,0.98)$ & $1.01(0.98,1.04)$ & 0.61 & 0.30 \\
\hline Tea (per $100 \mathrm{~g}$ ) & $0.99(0.97,1.02)$ & $1.00(0.96,1.03)$ & $1.00(0.96,1.03)$ & 0.75 & 0.84 \\
\hline Coffee $($ per $100 \mathrm{~g})$ & $0.99(0.97,1.01)$ & $0.98(0.97,0.98)$ & $1.01(0.99,1.03)$ & 0.28 & 0.34 \\
\hline \multicolumn{6}{|l|}{ Alcohol consumption (g/day $)^{\mathrm{b}}$} \\
\hline 0 & $1.55(1.26,1.89)$ & $1.32(1.24,1.41)$ & $1.17(0.95,1.44)$ & 0.15 & 0.41 \\
\hline$>0-6$ & 1 & 1 & 1 & & \\
\hline
\end{tabular}


Table 2 (continued)

\begin{tabular}{|c|c|c|c|c|c|}
\hline Lifestyle factor & Diabetic & Non-diabetic & Ratio & $p$ value difference & $p$ value interaction \\
\hline$>6-12$ & $0.85(0.67,1.09)$ & $0.80(0.75,0.85)$ & $1.07(0.83,1.37)$ & 0.60 & 0.81 \\
\hline$>12-24$ & $1.00(0.80,1.26)$ & $0.79(0.75,0.84)$ & $1.27(1.00,1.60)$ & 0.05 & 0.14 \\
\hline$>24-60$ & $1.05(0.83,1.32)$ & $0.87(0.82,0.93)$ & $1.20(0.94,1.53)$ & 0.15 & 0.49 \\
\hline$>60$ & $1.37(1.02,1.84)$ & $1.13(1.04,1.23)$ & $1.21(0.89,1.65)$ & 0.21 & 0.94 \\
\hline Continuous (per 12 g) & $0.95(0.92,0.99)$ & $0.94(0.93,0.95)$ & $1.02(0.98,1.06)$ & 0.36 & 0.86 \\
\hline \multicolumn{6}{|l|}{ Leisure-time physical activity ${ }^{a}$} \\
\hline Low & 1 & 1 & 1 & & \\
\hline Medium & $0.92(0.77,1.09)$ & $0.86(0.81,0.90)$ & $1.07(0.89,1.29)$ & 0.46 & 0.80 \\
\hline High & $0.86(0.71,1.03)$ & $0.88(0.83,0.93)$ & $0.98(0.81,1.18)$ & 0.80 & 0.25 \\
\hline Very high & $0.74(0.60,0.91)$ & $0.81(0.77,0.86)$ & $0.91(0.74,1.13)$ & 0.40 & 0.07 \\
\hline Continuous (per category) & $0.93(0.88,0.98)$ & $0.94(0.92,0.96)$ & $0.99(0.93,1.05)$ & 0.65 & 0.03 \\
\hline \multicolumn{6}{|l|}{ Smoking status ${ }^{\mathrm{a}}$} \\
\hline Never & 1 & 1 & 1 & & \\
\hline Former & $1.36(1.13,1.64)$ & $1.29(1.23,1.36)$ & $0.85(0.66,1.08)$ & 0.60 & 0.73 \\
\hline Current & $2.29(1.91,3.01)$ & $2.27(2.17,2.38)$ & $1.01(0.84,1.22)$ & 0.92 & 0.26 \\
\hline
\end{tabular}

${ }^{a}$ Model 1: age- and centre-stratified and adjusted for sex, prevalence of heart disease, cancer or stroke, educational attainment, diabetes medication use (in diabetic individuals), and the following when there were no exposure variables: alcohol consumption, smoking behaviour, physical activity and underlying dietary patterns

${ }^{\mathrm{b}}$ Model 1 additionally adjusted for alcohol consumption in the past

completely rule out the possibility that these differences are due to chance, since many differences were tested for. Assuming that the observed differences in associations are true differences, we hypothesise that they can be explained by the same underlying mechanisms as are involved in the prevention of diabetes. Fruits, legumes, nuts, seeds, pasta, poultry and vegetable oil are rich in vitamins, antioxidants, unsaturated fatty acids, polyphenols and fibre and low in saturated fat and sugars. These compounds may be responsible for improving endothelial function, blood pressure and blood lipids, as well as lowering oxidative stress, inflammation, insulin resistance and impaired glucose tolerance, and thus diabetes, CVD and overall mortality [32-36].

In conclusion, it appears that the intake of some food groups is more beneficial (fruits, legumes, nuts, seeds, pasta, poultry, vegetable oil) or more detrimental (soft drinks, butter, margarine, cake, cookies) with respect to mortality risk in people with diabetes. This may indicate that individuals with diabetes may benefit more from a healthy diet than people without diabetes. However, since the directions of association were generally the same, recommendations for a healthy diet should be similar.

Our study could be interpreted in the context of impaired glucose tolerance or undiagnosed diabetes. The clinical diagnosis of diabetes is defined by the level of hyperglycaemia, giving rise to risk of macro- and microvascular complications [13]. Diabetes can be considered a continuing process of declining glycaemic control, where individuals shift from health to impaired glucose tolerance, undiagnosed diabetes and finally diagnosed diabetes. It has been shown that glycaemic control is related to increasing risk of macrovascular complications throughout the whole range of concentrations, even below the diabetic threshold [14]. Impaired glucose tolerance has already been shown to be associated with increased CVD risk [37]. Even more complicated in this context, about $50 \%$ of all people with type 2 diabetes are believed to be undiagnosed [13]. Our findings highlight that these difficulties in recognising and diagnosing diabetes and its different stages are of minor importance with respect to healthy diet and lifestyle recommendations, because no difference in recommendations depending on the stage of the disease seems necessary.

Under-reporting of energy intake has been widely acknowledged in obese people, and it has also been reported that obese individuals with diabetes under-report their energy intake more than obese individuals without diabetes [38]. Indeed, a larger proportion of people with diabetes were classified as energy misreporters than those without diabetes. However, associations were not affected when those classified as energy misreporters were excluded. Energy adjustment seems to minimise the problems related to selective misreporting [20,39]. Moreover, although people with diabetes are supposed to undergo nutrition and health counselling, this was not reflected in their lifestyle behaviours in the present or other studies [40, 41].

This study benefitted from a large sample size, multicentric design, verification of diabetes diagnoses, wide range of variables and a long follow-up period. Moreover, multiple 
imputation of missing values and appropriate confounder selection and adjustment were used to reduce potential bias. Self-reports of diabetes at baseline were confirmed with a second information source, but when no additional information source was available, we were unable to discriminate true- from false-positive case classifications. Because no systematic screening was conducted, selection bias might have been introduced in different ways. For example, we cannot rule out the possibility that only the most advanced cases were classified as diabetes, or that people with a family history of diabetes or other concomitant comorbidities who have more contact with healthcare services were identified as having diabetes. Furthermore, we do not know whether the participants without diabetes at baseline have since developed the disease during follow-up. This could have reduced the external validity of our study. However, selective participation should not impair aetiological associations between a lifestyle factor and an outcome. Because aetiological associations were the main objective of the study, we do not think that the representativeness has biased the results.

In conclusion, we observed that diabetes status did not appear to influence the relations between most lifestyle factors and mortality risk. This study suggests that, with respect to mortality, lifestyle advice for people with diabetes should not differ from the existing recommendations for the general population. It may be that those with diabetes benefit more from a healthy diet than diabetes-free individuals. However, this has to be confirmed in further studies.

Acknowledgements The authors would like to thank W. Bernigau (German Institute of Human Nutrition, Potsdam-Rehbruecke, Germany) for help with statistical analysis.

Funding This study was supported by a European Foundation for the Study of Diabetes (EFSD)/Sanofi-Aventis grant. The sponsor did not have any role in the design and conduct of the study, collection, management, analysis and interpretation of the data, and preparation, review, or approval of the manuscript.

Duality of interest The authors declare that there is no duality of interest associated with this manuscript.

Contribution statement DS designed the study, acquired the data, performed statistical analysis, interpreted the data, drafted the article, and approved the final version to be published. UN and HB designed the study, acquired the data, drafted, reviewed and critically revised the article, and approved the final version to be published. All other authors acquired the data, reviewed and critically revised the article, and approved the final version to be published.

\section{References}

1. Deshpande AD, Harris-Hayes M, Schootman M (2008) Epidemiology of diabetes and diabetes-related complications. Phys Ther 88:1254-1264
2. Sarwar N, Gao P, Seshasai SR et al (2010) Diabetes mellitus, fasting blood glucose concentration, and risk of vascular disease: a collaborative meta-analysis of 102 prospective studies. Lancet 375:22152222

3. Seshasai SR, Kaptoge S, Thompson A et al (2011) Diabetes mellitus, fasting glucose, and risk of cause-specific death. N Engl J Med 364: 829-841

4. Berry J, Keebler ME, McGuire DK (2004) Diabetes mellitus and cardiovascular disease. Pandora's box has been opened. Herz 29: 456-462

5. Buse JB, Ginsberg HN, Bakris GL et al (2007) Primary prevention of cardiovascular diseases in people with diabetes mellitus: a scientific statement from the American Heart Association and the American Diabetes Association. Diabetes Care 30:162-172

6. IDF Clinical Guidelines Task Force (2005) Global guideline for type 2 diabetes. International Diabetes Federation, Brussels

7. Lichtenstein AH, Appel LJ, Brands M et al (2006) Summary of American Heart Association Diet and Lifestyle Recommendations revision 2006. Arterioscler, Thromb, Vasc Biol 26:2186-2191

8. Sluik D, Boeing H, Montonen J et al (2011) Associations between general and abdominal adiposity and mortality in individuals with diabetes mellitus. Am J Epidemiol 174:22-34

9. Sluik D, Boeing H, Bergmann MM et al (2012) Alcohol consumption and mortality in individuals with diabetes mellitus. Br J Nutr 108: $1307-1315$

10. Sluik D, Buijsse B, Muckelbauer R et al (2012) Physical activity and mortality in individuals with diabetes mellitus: a prospective study and meta-analysis. Arch Intern Med 172:1285-1295

11. Clark CM Jr (2000) Combating sloth as well as gluttony: the role of physical fitness in mortality among men with type 2 diabetes. Ann Intern Med 132:669-670

12. American Diabetes Association (2011) Standards of medical care in diabetes - 2011. Diabetes Care 34(Suppl 1):S11-S61

13. Ryden L, Standl E, Bartnik M et al (2007) Guidelines on diabetes, pre-diabetes, and cardiovascular diseases: executive summary. The Task Force on Diabetes and Cardiovascular Diseases of the European Society of Cardiology (ESC) and of the European Association for the Study of Diabetes (EASD). Eur Heart J 28:88-136

14. Khaw KT, Wareham N, Luben R et al (2001) Glycated haemoglobin, diabetes, and mortality in men in Norfolk cohort of European Prospective Investigation of Cancer and Nutrition (EPIC-Norfolk). BMJ 322:15-18

15. Riboli E, Hunt KJ, Slimani N et al (2002) European Prospective Investigation into Cancer and Nutrition (EPIC): study populations and data collection. Public Health Nutr 5:1113-1124

16. Ocke MC, Bueno-de-Mesquita HB, Pols MA, Smit HA, van Staveren WA, Kromhout D (1997) The Dutch EPIC food frequency questionnaire. II. Relative validity and reproducibility for nutrients. Int J Epidemiol 26(Suppl 1):S49-S58

17. Riboli E, Elmstahl S, Saracci R, Gullberg B, Lindgarde F (1997) The Malmo Food Study: validity of two dietary assessment methods for measuring nutrient intake. Int J Epidemiol 26(Suppl 1):S161-S173

18. Bohlscheid-Thomas S, Hoting I, Boeing H, Wahrendorf J (1997) Reproducibility and relative validity of food group intake in a food frequency questionnaire developed for the German part of the EPIC project. European Prospective Investigation into Cancer and Nutrition. Int J Epidemiol 26(Suppl 1):S59-S70

19. Kaaks R, Riboli E (1997) Validation and calibration of dietary intake measurements in the EPIC project: methodological considerations. European Prospective Investigation into Cancer and Nutrition. Int J Epidemiol 26(Suppl 1):S15-S25

20. Willett WC, Howe GR, Kushi LH (1997) Adjustment for total energy intake in epidemiologic studies. Am J Clin Nutr 65:1220S-1228S, discussion 1229S-1231S

21. Slimani N, Ferrari P, Ocke M et al (2000) Standardization of the 24-hour diet recall calibration method used in the European 
Prospective Investigation into Cancer and Nutrition (EPIC): general concepts and preliminary results. Eur J Clin Nutr 54:900-917

22. Klipstein-Grobusch K, Slimani N, Krogh V et al (2002) Trends in self-reported past alcoholic beverage consumption and ethanol intake from 1950 to 1995 observed in eight European countries participating in the European Investigation into Cancer and Nutrition (EPIC). Public Health Nutr 5:1297-1310

23. Cox DR, Oakes D (1984) Analysis of survival data. Chapman and Hall, London

24. Lunn M, McNeil D (1995) Applying Cox regression to competing risks. Biometrics 51:524-532

25. Hoffmann K, Pischon T, Schulz M, Schulze MB, Ray J, Boeing H (2008) A statistical test for the equality of differently adjusted incidence rate ratios. Am J Epidemiol 167:517-522

26. Greenland S, Pearl J, Robins JM (1999) Causal diagrams for epidemiologic research. Epidemiology 10:37-48

27. Sterne JA, White IR, Carlin JB et al (2009) Multiple imputation for missing data in epidemiological and clinical research: potential and pitfalls. BMJ 338:b2393

28. Newgard CD, Haukoos JS (2007) Advanced statistics: missing data in clinical research-part 2: multiple imputation. Acad Emerg Med 14: $669-678$

29. Yuan YCY (2000) Data analysis papers: multiple imputation for missing values: concepts and new development. SAS Institute Inc, SUGI Proceedings

30. Batty GD, Shipley MJ, Marmot M, Smith GD (2002) Physical activity and cause-specific mortality in men with type 2 diabetes/ impaired glucose tolerance: evidence from the Whitehall study. Diabet Med 19:580-588

31. Ford ES, DeStefano F (1991) Risk factors for mortality from all causes and from coronary heart disease among persons with diabetes.
Findings from the National Health and Nutrition Examination Survey I Epidemiologic Follow-up Study. Am J Epidemiol 133:1220-1230

32. Alexiadou K, Katsilambros N (2011) Nuts: anti-atherogenic food? Eur J Intern Med 22:141-146

33. Ness AR, Powles JW (1997) Fruit and vegetables, and cardiovascular disease: a review. Int J Epidemiol 26:1-13

34. Soinio M, Laakso M, Lehto S, Hakala P, Ronnemaa T (2003) Dietary fat predicts coronary heart disease events in subjects with type 2 diabetes. Diabetes Care 26:619-624

35. Tanasescu M, Cho E, Manson JE, Hu FB (2004) Dietary fat and cholesterol and the risk of cardiovascular disease among women with type 2 diabetes. Am J Clin Nutr 79:999-1005

36. Venn BJ, Mann JI (2004) Cereal grains, legumes and diabetes. Eur J Clin Nutr 58:1443-1461

37. Ford ES, Zhao G, Li C (2010) Pre-diabetes and the risk for cardiovascular disease: a systematic review of the evidence. J Am Coll Cardiol 55:1310-1317

38. Salle A, Ryan M, Ritz P (2006) Underreporting of food intake in obese diabetic and nondiabetic patients. Diabetes Care 29:2726-2727

39. Sluijs I, van der Schouw YT, van der A D (2010) Carbohydrate quantity and quality and risk of type 2 diabetes in the European Prospective Investigation into Cancer and Nutrition-Netherlands (EPIC-NL) study. Am J Clin Nutr 92:905-911

40. Nelson KM, Reiber G, Boyko EJ (2002) Diet and exercise among adults with type 2 diabetes: findings from the Third National Health and Nutrition Examination Survey (NHANES III). Diabetes Care 25: $1722-1728$

41. Nothlings U, Boeing H, Maskarinec G et al (2011) Food intake of individuals with and without diabetes across different countries and ethnic groups. Eur J Clin Nutr 65:635-641 